\title{
ÜBER FINNLANDS REZENTE UND SUBFOSSILE DIATOMEEN V
}

\author{
Karl Mölder und Risto Tynni
}

\begin{abstract}
Mölder, Karl und Tynni, Risto 1971: Über Finnlands rezente und subfossile Diatomeen V. Bull. Geol. Soc. Finland 43, 203-220.

The article deals with the distribution and ecology of the Thalassionema, Asterionella, Amphicampa, Peronia, Eunotia and Actinella species known in Finland.

Karl Mölder, Kelohongantie 2 C 21, Tapiola, Finnland.

Risto Tynni, Geologische Forschungsanstalt in Finnland, Otaniemi, Finnland.
\end{abstract}

\section{Thalassionema}

Linear längliche, schmale Schalen, in deren Valvarebene am Rande kleine Randdörnchen sitzen, an den apikalen Enden ist das Randdörnchen auswärts gerichtet.

Die Schalen bilden sternförmige oder zickzackförmige Kolonien. In Finnland ist nur die Art $T$. nitzschioides anzutreffen.

\section{Thalassionema nitzschioides Grunow}

Synonym: Thalassiotbrix nitzschioides Grunow

Planktonform von Salz- und Brackwasser, die an der Küste des Finnischen und des Bottnischen Meerbusens sowie im offenen Meer verhältnismässig selten vorkommt. Subfossil ist ihr Fortbestehen in Sedimenten sehr unregelmässig.

\section{Asterionella}

Längliche und schmale, an den Enden im allgemeinen erweiterte Schalen, die eine schmale Pseudoraphe aufweisen. Die Schalen bilden im allgemeinen sternförmige Kolonien.

\section{Asterionella formosa Hassal}

In Süsswasser wachsende Planktonform, u.a. in vielen eutrophen Seen zeitweilig vorherr- schende Art. Sowohl in Süd- als auch in Nordfinnland allgemein anzutreffen. Es ist denn auch festgestellt worden, dass die Art wenigstens in den Temperaturgrenzen von $1.5-24^{\circ} \mathrm{C}$ wächst, im See Tornionjärvi sogar bei $0.5^{\circ} \mathrm{C}$ (Lund 1950), und sie gehört zu den sog. eurythermen Diatomeen. Sie vermag Phosphor über ihren unmittelbaren Bedarf zu speichern (Lund op. cit.), und der Phosphorverbrauch liegt maximal im Gebiet $\mathrm{pH}$ 6-7, weswegen Mackereth (1953, nach Cholnoky 1968) für die Art das Optimum als pH-Gebiet 6-7 dargestellt hat. Man kennt sie auf Grund ihres Vorkommens als alkaliphil (Hustedt 1957, Cholnoky op. cit.).

Var. acearoides Lemmermann

Seltene Variation, die an vier Stellen in Südund Nordfinnland angetroffen worden ist.

Asterionella gracillima (Hantzsch) Heiberg

Synonym: Asterionella formosa var. gracillima Grunow

In Finnland eine ungefähr gleich häufige Art wie $A$. formosa, aber der Schwerpunkt des Vorkomens liegt südlicher. Im Schrifttum ist die Art oft als alkaliphil angegeben, aber wir haben sie in einigen Kleinseen mit $\mathrm{pH} 5-6$ als häufig. festgestellt. 


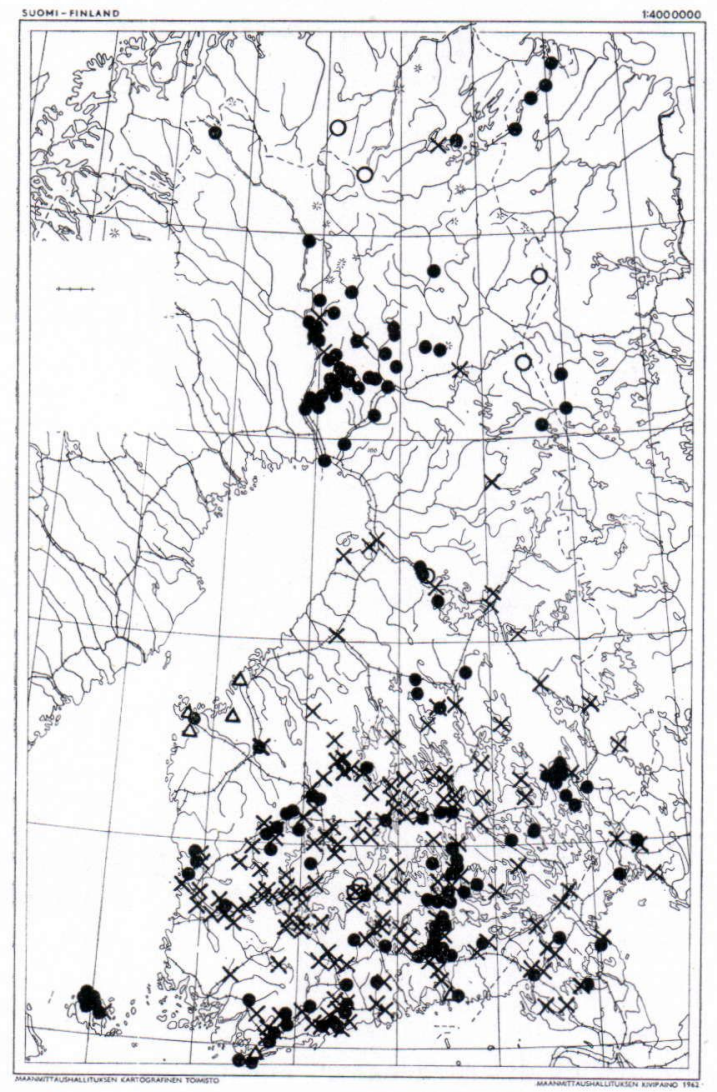

Abb. 1. Rezente Verbreitung von Thalassionema nitzscbioides (Dreiecke), Asterionella formosa (Punkte), A. gracillima (Kreuze) und Amphicampa bemicyclus (Ringe).

\section{Asterionella ralfsii W. Smith}

Synonym: Peronia erinacea Brébisson \& Arnott

Süsswasserform, seltener als die vorhergehenden. Im Schrifttum wird sie oft Peronia erinacea genannt. Azidophile Form, die u.a. in Moortümpeln wächst.

\section{Amphicampa}

Stark gekrümmte schmale Schalen, in deren Valvarebene nur eine quere Liniierung zu sehen ist. Pseudoraphe und Raphe fehlen, in welcher Hinsicht sie sich wesentlich von der Gattung Eunotia unterscheidet.

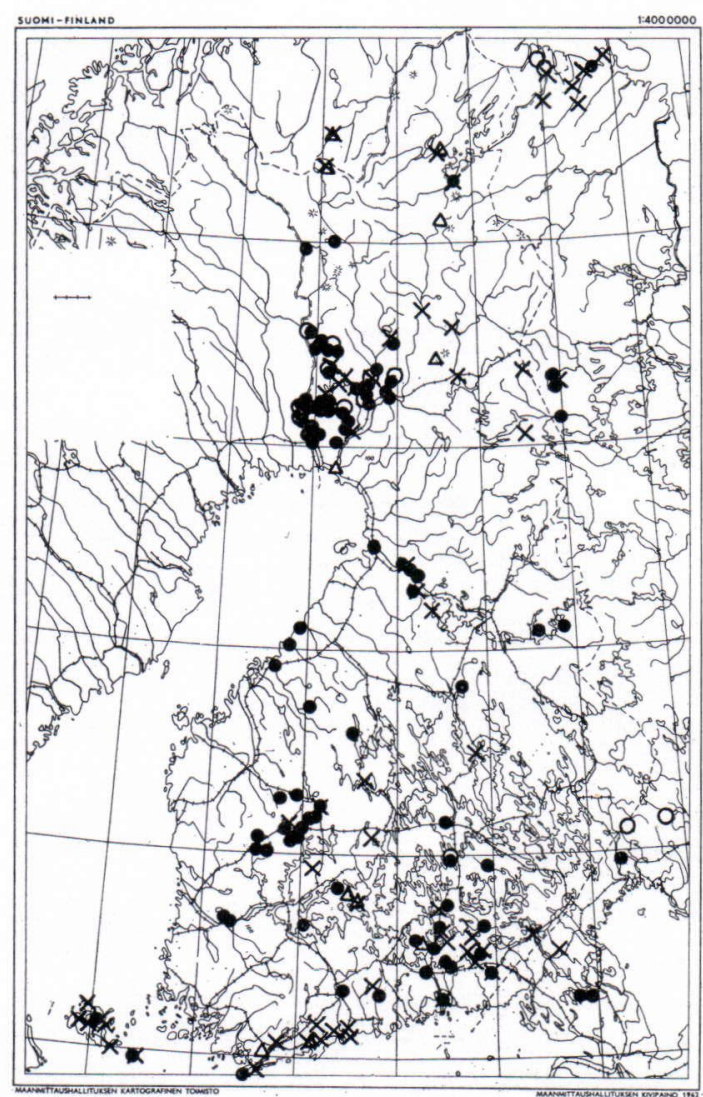

Abb. 2. Rezente Verbreitung von Peronia beribaudi (Ringe), Eunotia alpina (Punkte), E. arcus (Kreuze) und $E$. bactriana (Dreiecke).

\section{Amphicampa bemicyclus (Ehr.) Karsten}

Synonyme: Pseudoeunotia bemicyclus Grun., Semiorbis bemicyclus (Ehr.) Patr.

Form eines kleinen, kühlen und humushaltigen Süssgewässers. Auch in Finnland ist festzustellen, dass die Art unter ähnlichen Verhältnissen wie Tabellaris binalis gedeiht (vgl. Kolbe \& Silfversparre 1950).

\section{Peronia}

Die Schale ähnelt Asterionella. Sie zeigt eine schmale Pseudoraphe, aber ausserdem an den Enden der einen Schalenhälfte kurze Raphenäste. Nur eine Art ist bekannt. 
Peronia heribaudi Brun \& Peragallo

Epiphytische Süsswasserart, die in Nord- und Südfinnland in einigen Seen und Moortümpeln angetroffen worden ist. Azidophile Form.

\section{Eunotia}

Längliche, mehr oder weniger bogenförmig gekrümmte Schalen, die transapikal liniiert oder punktliniiert sind. Die Raphenäste in den apikalen Enden kurz, die Endknoten allgemein deutlich. Dagegen sind Zentralknoten allgemein nicht vorhanden oder nicht zu sehen. Jedenfalls liegen bisweilen weiter nach der Mitte zu an den Ventralseiten kleine Höcker, die den ZentralknotenAnsätzen entsprechen. Eine schmale Pseudoraphe verläuft ausserdem nahe der Ventralseite. Dorsalseits haben manche Arten hintereinandergelegene grosse Höcker.

Der grösste Teil der weiter unten folgenden Arten ist in seinen ökologischen Ansprüchen gleichartig. Es sind Formen süss-saurer und kühler Kleingewässer. Viele Arten gedeihen auch an feuchten Felswänden sowie als Epiphyten an Moosen. Sie wachsen einzeln oder mit ihren Seiten aneinander befestigt in Kolonien. Die Eunotia-Arten gedeihen nicht besonders in fliessendem oder alternativ in nährstoffhaltigem Wasser (Round 1959). Übermässiger Nährstoffgehalt dürfte ein beschränkender Faktor sein, denn viele Arten kommen in Quellen und in Lapplands kargen, reinwässerigen Bächen reichlich vor. Besonders im Gebiet Lapplands sind die Eunotia-Arten häufig anzutreffen.

Subfossil findet sich Eunotia oft zusammen mit Pinnularia in den basalen Sedimenten warviger Tone, in denen die Diatomeendichte im Mittel sehr gering ist. Ihrer Herkunft nach können diese in den Bereich des Inlandeises zurückführen oder zeitlich und räumlich sonstwie sekundär sein. In als primär anzusehenden Zusammenhängen bezeugen die Eunotia-Arten in den Sedimenten eine flache Süsswasserphase oder ufernahe Sedimentationsverhältnisse.

\section{Eunotia alpina (Naeg.) Hustedt}

Eine der E. lunaris ähnliche Art, aber noch schmäler als sie. Rezente Beobachtungen verhältnismässig reichlich. Azidophile Form (Foged 1958, 1964, Meriläinen 1969).

\section{Eunotia angusta (Grun.) Å. Berg}

Eine der E. parallela ähnliche Art, die von Cleve-Euler (1953) aus Finnisch-Lappland dargestellt worden ist.

\section{Eunotia arcus Ehrenberg}

Synonym: Eunotia arcus var. minor Grun.

Verhältnismässig häufig in Süd- und Nordfinnland, besonders in kleinen Weihern. Nach Hustedt wächst sie auch in Kalkgegenden. Alkaliphile Form.

Fo. plicata (Brun) Hèrib.

Diese seltene Form ist aus Lappland dargestellt (Cleve-Euler 1953).

\section{Var. bidens Grunow}

Kommt zuweilen neben der Hauptform vor.

Var. fallax Hustedt

Synonym: Eunotia arcus var. subalpina Fontell

Rezent selten, aber subfossil rel. häufige Variation. Hauptvorkommen mit Moortümpeln verbunden.

\section{Var. oxycephala Meister}

Cleve-Euler (1953) hat diese Variation im Grundschlamm des Sees Inarijärvi angetroffen.

\section{Var. uncinata Grunow}

In Lappland selten vorkommende Variation.

\section{Eunotia attenuata A. Cleve 1934}

Kürzere Form als die 1940 von Patrick aus Brasilien dargestellte. Nur in Lappland festgestellte Eunotia-Art. (Cleve-Euler 1953). 


\section{Eunotia bactriana Ehrenberg}

In Lappland wie auch Südfinnland gefundene Art kalten, humushaltigen Wassers.

\section{Var. bisinuosa A. Cleve}

Cleve-Euler (1953) hat die Variation aus einem Moor am See Kuolajärvi, Lappland, mitgeteilt.

\section{Eunotia bidentula W. Smith}

Ähnelt E. diodon, unterscheidet sich aber von ihr vorzugsweise durch die geradlinigere Ventralseite. Die Art in Lappland und Südfinnland festgestellt. Nach Hustedt und Krasske (1949) eine nordische bzw. alpine Art oder eine Form rel. kalten Wassers.

\section{Eunotia bigibba Kützing}

Synonym: Eunotia sarekensis var. bigibba (Kütz.) A. Berg

Eine der E. suecica (E. sarekensis) ähnliche, aber schmälere, zweihöckerige Art. Sie ist in Süd- und Nordfinnland rel. selten angetroffen worden. Nach Hustedt ist sie eine Charakterart feuchter Felsen.

\section{Var. pumila Grunow}

Synonym: Eunotia sarekensis var. pumila (Grun.) ^. Berg

Kleiner als die Hauptart und ebenso selten wie diese.

\section{Eunotia clevei Grunow}

Eine der grössten Eunotia-Arten, die auch ökologisch eine Ausnahme bildet. Sie wächst in grossen Seen, u.a. im Ladoga, Kemijärvi und Lohjanjärvi. Sie gedeiht in nährstoffreichem Wasser und ist wahrscheinlich alkaliphil. Die subfossilen Vorkommen verbinden sich mit Grossseephasen, insbesondere mit Litoralsedimenten des Ancylussees.

\section{Eunotia crista-galli Cleve}

Seltene Art, die in Finnland in Torhola ( $\mathrm{Hu}-$ stedt), Virroinen (Mölder) und in Lappland in einigen Tümpeln (Krasske) festgestellt worden ist.

\section{Eunotia denticulata (Brèb.) Rabenhorst}

Synonym: Eunotia denticulata var. borealis A. Cleve

Krasske (1949) hat die Art in Westlappland an Moosen und in Moortümpeln gefunden, CleveEuler (1953) in einigen Gewässern Lapplands. Die Art ist häufig in einem kleinen See von Utsjoki, der winters trocken ist. Die südlichste Beobachtung ist bei Meriläinen (1969) von einem humushaltigen kleinen Weiher in Savo. Kaltwasserform.

Var. fennica Hustedt.

Schalen gestreckter als bei voriger. Hustedt hat sie aus Sphagnumtümpeln am Ostfuss des Vuokatti festgestellt.

\section{Eunotia diodon Ehrenberg}

Synonyme: Eunotia diodonf. minor Grun., E. d. var. diminuta Grun..

In Lappland und Südfinnland besonders in kleinen Weihern wachsende Form kühlen Wassers, nach Krasske (1949) häufige Art auf feuchten Felsen des Jehkastunturi.

\section{Eunotia elegans Östrup}

Synonym: Eunotia voluta $\AA$. Berg

Sehr seltene Art, in einigen Kleinseen Nordund Südfinnlands festgestellt.

\section{Eunotia exigua (Brèb.) Rabenhorst}

Synonyme: Eunotia paludosa Grun., E. exigua var. nymanniana Grun., E. nymanniana var. genuina, var. inflata A. Mayer.

Häufige Art besonders in Moortümpeln. Azidobionte Form.

\section{Var. bidens Hustedt}

Nach Krasske (1949) auf dem Jehkastunturi auf feuchten Felsen.

Var. compacta Hustedt

Synonym: Eunotia nymanniana var. compacta (Hust.) A. Cleve

Besonders in einigen Moortümpeln in Lappland häufige Variation. 


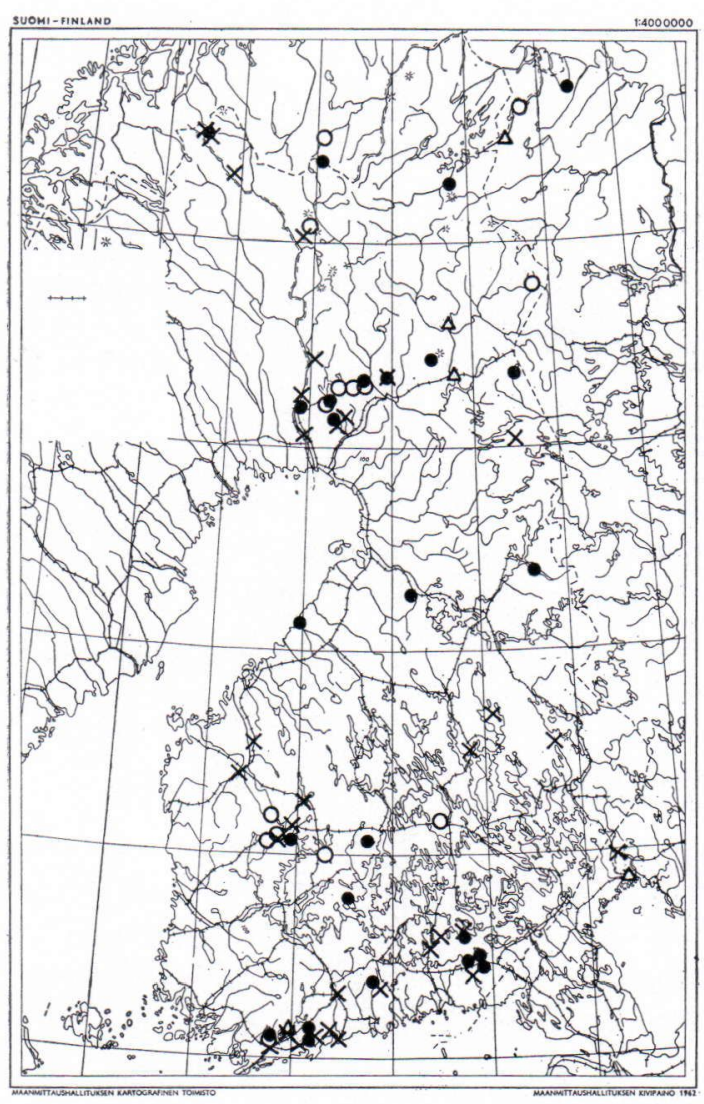

Abb. 3. Rezente Verbreitung von Eunotia bidentula (Punkte), E. bigibba (Ringe), E. clevei (Dreiecke) und E. diodon (Kreuze).

\section{Eunotia faba (Ehr.) Grunow}

Verhältnismässig häufige azidophile Art, die u.a. in Moortümpeln und Quellen wächst.

\section{Eunotia fallax A. Cleve}

Kleine, schmale, entfernt gestreifte Art, deren Enden in dorsaler Richtung gekrümmt sind. Krasske (1949) und Cleve-Euler (1953 var. lapponica) haben die Art aus Lappland dargestellt. Nach Hustedt wächst sie in Mooren, Quellen und an feuchten Felswänden.

\section{Var. dispersa A. Cleve}

An ihren Enden verschmälerte Form, von Cleve-Euler für den Fluss Vaskojoki und den Teich Haukilampi in Petsamo belegt. Möglicher-

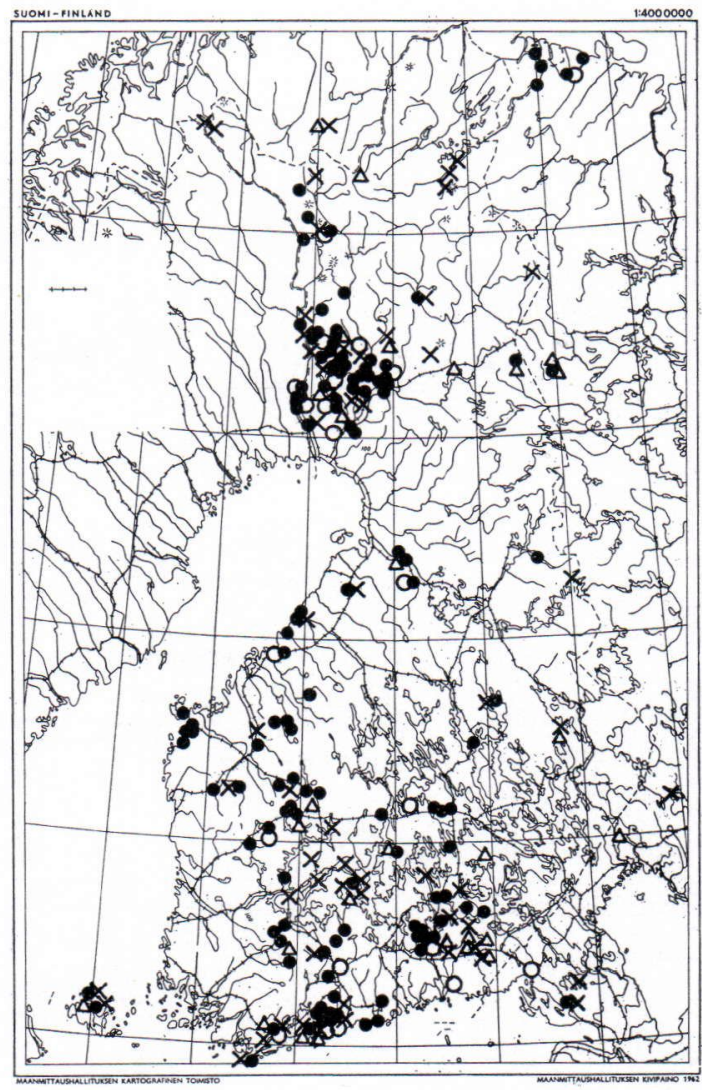

Abb. 4. Rezente Verbreitung von Eunotia exigua (Punkte), E. faba (Kreuze), E. fallax (Ringe) und E. flexuosa (Dreiecke).

weise ist die Variation mit der folgenden zu vereinigen.

\section{Var. gracillima Krasske}

Schmälere Form als die vorhergehende, ähnelt auch $E$. lunaris var. subarcuata, ist aber entfernt gestreift. In Nord- und Südfinnland selten angetroffen.

\section{Eunotia flexuosa (Brèb.) Kützing}

Synonyme: Eunotia flexuosa var. pachycephala, var. eurycephala, var.? bicapitata Grunow, E. biceps var. genuina, var. bicapitata A. Mayer

Besonders in kleinen Weihern, Moortümpeln und Quellen epiphytisch wachsende azidophile Art. 


\section{Eunotia formica Ehrenberg}

Vielgestaltige Art. Rezent relativ selten, aber subfossil in vielen Spät- und Postglazialsedimenten von Kleinseebecken häufig. In bezug auf $\mathrm{pH}$ indifferent.

\section{Eunotia gracilis (Ehr.) Rabenhorst}

Synonym: Eunotia glacialis Meister

Verhältnismässig seltene Art, die besonders in kühlen, humushaltigem und mineralstoffarmem Wasser vorkommt.

\section{Var. fennoscandica (A. Cl.)}

Synonym: Eunotia glacialis var. fennoscandica A. Cl.

Cleve-Euler (1953) hat sie aus Kemijärvi dargestellt. Subfossil in Lappland häufiger.

\section{Eunotia byberborea $\AA$. Berg}

Subfossil in den Sedimenten vom Weiher Laenlampi in Korvala, Lappland.

\section{Eunotia kocheliensis O. Müller}

Nur subfossil selten festgestellte Art.

\section{Eunotia lapponica Grunow}

Einige Beobachtungsstellen in Nord- und Südfinnland. Mag jedoch verhältnismässig häufig in Lappland in Lachen, Mooren und Quellen vorkommen. Azidophile Kaltwasserform.

\section{Eunotia lunaris (Ehr.) Grunow}

Häufige Eunotia-Art. Sie ist ein Epiphyt, kann aber aúch im Plankton verhältnismässig eutropher Seen zeitweilig reichlich vorkommen. Nach Cholnoky (1970) sind derartige Vorkommen sekundär, denn die Art ist eine Form sauren oligotrophen Wassers, die einen Anstieg oder Wechsel des osmotischen Druckes nicht verträgt. Ihr Vermögen, sich von Strömungen treiben zu lassen, wird auch dadurch erwiesen, dass sie ausnahmsweise in Flüssen angetroffen wird (Round 1959, Patrick \& Reimer 1966).
Var. capitata Grunow

Neben der vorhergehenden besonders in Lappland rel. häufig.

Var. subarcuata (Naeg.) Grunow

Verhältnismässig häufige Variation, die allgemein neben der Hauptart auftritt.

Eunotia meisteri Hustedt

Seltene, kleine, azidophile Art.

Eunotia microcephala Krasske

Synonym: Eunotia perpusilla var. simplex (May) A. Berg

In Finnland nur in Lappland angetroffene azidophile Form kalten Wassers.

Var. tridentata (A. Mayer) Hustedt

In Lappland vier Beobachtungsstellen, u.a. hat Krasske (1949) sie neben der Hauptform in einer Lache auf dem Berg Muotkantakanvaara und im klarwässerigen Haukijärvi an Moosen angetroffen.

\section{Eunotia monodon Ehrenberg}

Grosse Eunotia-Art, rezent verhältnismässig selten, u.a. in vermoorten Weihern besonders in Nordfinnland. Kaltwasserform. Subfossil ist sie relativ häufig.

\section{Var. alpina Kützing}

Zwergform der vorherigen, von Cleve-Euler aus Westlappland und Ivalo dargestellt.

\section{Var. bidens (Greg.) W. Smith}

Synonyme: Eunotia jemtlandica Fontell, E. tibia var. bidens (Greg.) A. Cl.

Seltener als die Hauptart, azidophile Kaltwasserform. Besonders in kleinen Weihern.

Var. maior (W. Smith) Hustedt

Synonym: Eunotia maior Rabenhorst

Oft im Zusammenhang mit der Hauptform auftretende Variation. 


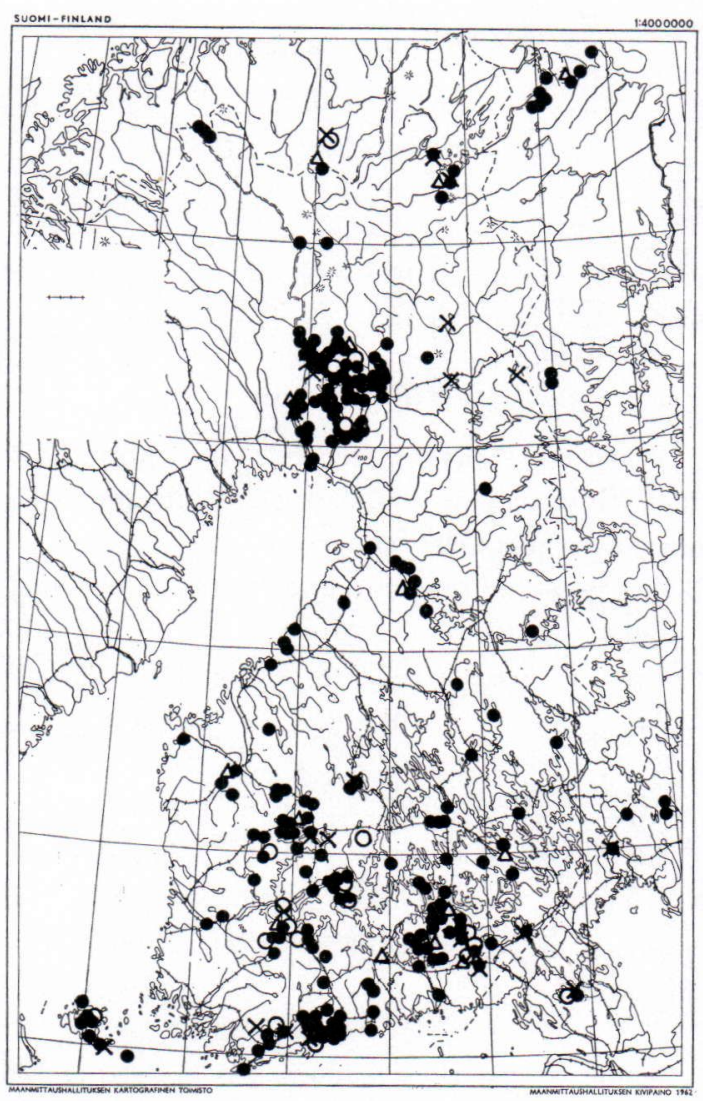

Abb. 5. Rezente Verbreitung von Eunotia formica (Ringe), E. gracilis (Kreuze), E. lapponica (Dreiecke) und E. lunaris (Punkte).

\section{Eunotia papilio (Grun.) Hustedt}

Synonyme: Himantidium papilio Ehr., Eunotia robusta var. papilio Grun., E. sarekensis var. papilio (Ehr.) Å. Berg.

Seltene Eunotia-Art. Nordisch-alpine Art, u.a. auf dem Saanatunturi in einem Bach an Moosen (Krasske 1949). Subfossil verhältnismässig häufig.

\section{Eunotia parallela Ehrenberg}

Synonyme: Eunotia parallela var. robusta, var. densestriata Fontell, E. angustata var. robusta, var. densestriata (Fontell) A. Bg., E. p. var. pseudoparallela A. Cl.

Besonders auf wässerigen Sphagnum-Mooren angetroffene Kaltwasserform.

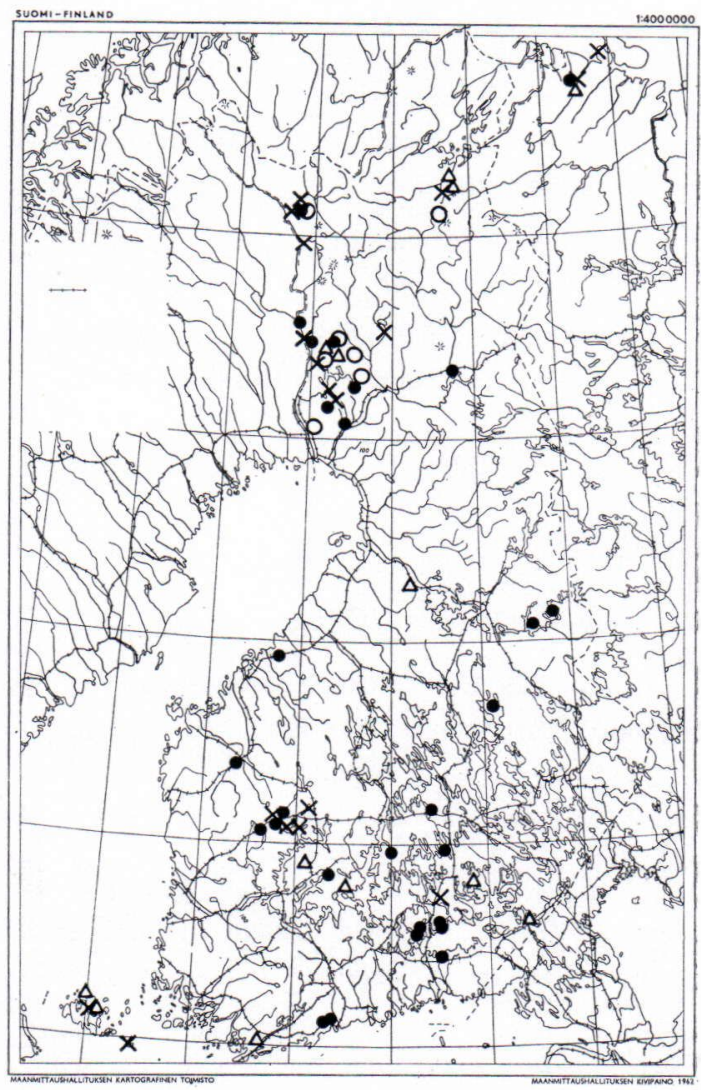

Abb. 6. Rezente Verbreitung von Eunotia meisteri (Punkte), E. microcephala (Ringe), E. monodon (Kreuze) und E. parallela (Dreiecke).

\section{Var. minor Mölder}

Beträchlich kleiner als die Hauptart. Aus Petsamo (Mölder 1937) sowie von Aland dargestellt (Mölder 1946 b).

\section{Eunotia pectinalis Rabenhorst}

Weitverbreitet häufige Eunotia-Art. Wächst in Weihern, Bächen, Quellen. Optimum-pH nach Cholnoky (1970) 6.5 (in Südafrika), aber die Art wächst auch in alkalischen Gewässern (u.a. Pork 1970).

Var. minor (Kütz.) Rabenhorst

Variation häufiger als die Hauptart. Azidophile Form. 
Fo. impressa (Ehr.) Hustedt

Synonym: Eunotia impressa var. angusta Grunow.

Kommt oft in gleichem Zusammenhang wie die Variation vor.

Fo. intermedia Krasske

Der E. faba sehr ähnliche Form, aber an den Enden schmäler. Seltene azidophile Form.

Var. undulata Ralfs

Sehr seltene Variation.

Var. ventralis (Ehr.) Hustedt

Oft in gleichen Zusammenhängen wie die Hauptart auftretende Variation.

\section{Eunotia polydentula Brun}

Synonym: Eunotia tridentata Ehrenberg

Kleine Eunotia-Art, die bes. in Moortümpeln, Quellen und an Moosen wächst.

\section{Var. perpusilla Grunow}

Synonym: Eunotia perpusilla var. genuina A. Cl.

Seltene Variation, schmäler als die vorige.

\section{Eunotia polyglyphis Grunow}

Verhältnismässig häufig vorwiegend in kleinen Weihern mit Moorufern. Form kühlen, humushaltigen Wassers.

\section{Eunotia praerupta Ehrenberg}

Eine der häufigsten Eunotia-Arten besonders in Lappland. Wächst in Weihern, Bächen und Quellen. Bezüglich pH azidophil oder neutral. Häufig in spät- oder postglazialen Süsswasserablagerungen.

Var. bidens (W. Sm.) Grunow

Seltenere Variation als die vorhergehende. Azidophil.
Var. inflata Grunow

Neben der Hauptart allgemein vorkommende Variation.

Var. muscicola Petersen

Kleine Form, die in Finnland nur in Lappland festgestellt worden ist.

Eunotia pseudopectinalis Hustedt

Von der Art E. pectinalis in erster Linie dadurch unterschieden, dass das Ende der Raphe mit einem zurücklaufenden Anhang versehen ist. Bisher nur in Lappland und Nordkarelien festgestellte Art, u.a. an Moosen in Bächen und Lachen (Hustedt 1962, Krasske 1949, CleveEuler 1953, Meriläinen 1967).

Eunotia reflexa fo. minor Hustedt

An Eunotia meisteri erinnertnde Form, Meriläinen (1969) hat sie aus dem meromikten See Valkiajärvi, Ruovesi, dargestellt.

\section{Eunotia robusta Ralfs}

Synonyme: Eunotia serra Ehr., E. robusta var. polyodon (Ehr.) Meist.

Verhältnismässig häufig in vermoorenden Tümpeln Süd- und Nordfinnlands. Azidophile, nordisch-alpine Form.

Var. diadema (Ehr.) Ralfs

Synonym: Eunotia diadema Ehrenberg.

Azidophile Form, häufiger als die vorhergehende. Wächst u.a. auf Sphagnum-Mooren.

Var. tetraodon (Ehr.) Ralfs

Synonyme: Eunotia diadema var. tetraodon (Ehr.) A. Cl., E. serra var. diadema (Ehr.) Patr.

Allgemeinster Vertreter der Art. Oft an denselben Stellen wie Var. diadema.

Eunotia septentrionalis Östrup

In Nordfinnland häufiger als in Südfinnland. Wächst besonders in Lachen und kleinen Bächen an Moosen. Azidophile Form kühlen Wassers. 


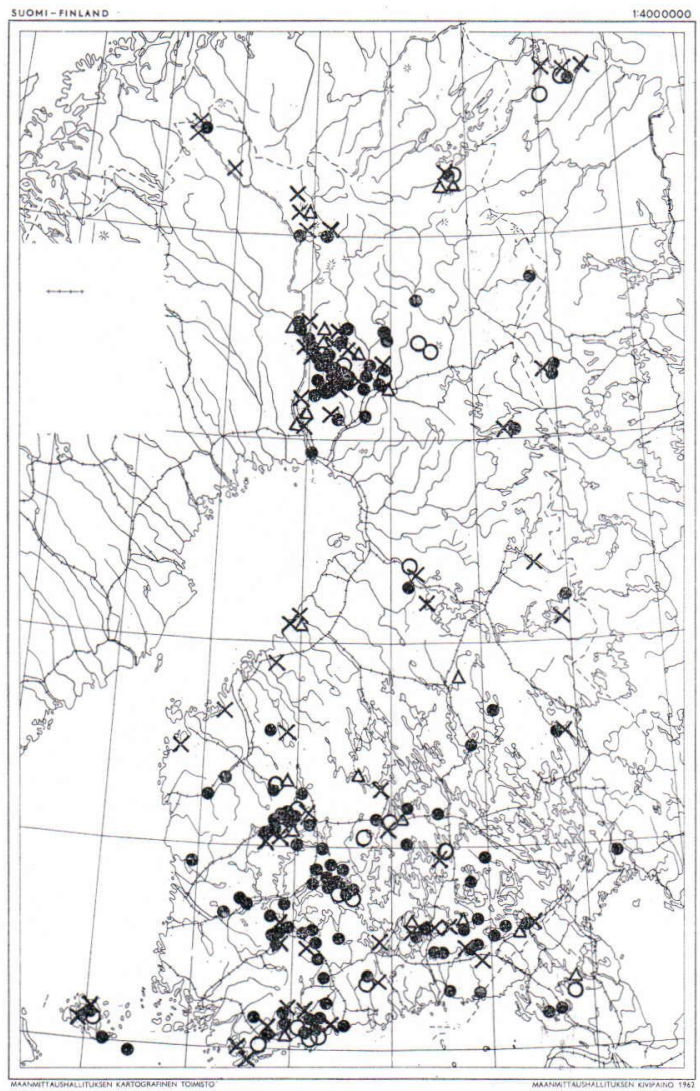

Abb. 7. Rezente Verbreitung von Eunotia pectinalis (Punkte), E. polydentula (Dreiecke), E. polyglyphis (Ringe) und E. praerupta (Kreuze).

\section{Var. bidens Hustedt}

Hustedt (1962) hat die Variation aus einem südfinnischen Weiher, dem Tuohilampi, dargestellt.

\section{Eunotia sibirica Cleve}

Rezent sehr seltere Art. Angetroffen in Kuorevesi, Mänttä (Mölder), Särkilampi, Südfinnland (Hustedt). Östliche Kaltwasserform. Subfossile Beobachtungen verhältnismässig häufig.

\section{Eunotia sudetica O. Müller}

Synonyme: Eunotia pectinalis var. crassa O. Müller, E. sudetica var. crassa (O. M.) A. Bg.

Wächst in Quellen und humushaltigen kleinen Gewässern. Azidophile Form.

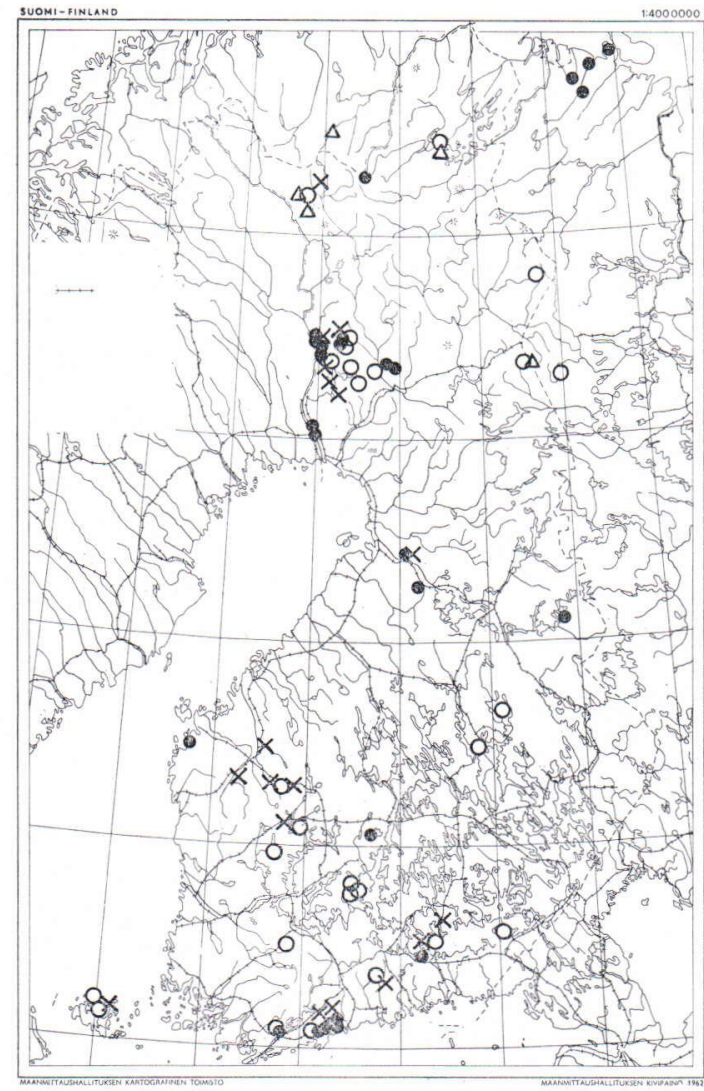

Abb. 8. Rezente Verbreitung von Eunotia pseudopectinalis (Dreiecke), E. robusta (Ringe), E. septentrionalis (Punkte) und $E$. sudetica (Kreuze).

Var. bidens Hustedt

Rezent nur in Westlappland, Pello, festgestellt, sowie im Flusse Käkkäläjoki (Cleve-Euler).

\section{Eunotia suecica A. Cleve}

Synonym: Eunotia sarekensis (A. Berg) A. Cl.

Eine in Finnland nur in Lappland festgestellte seltene Art. Wächst an Bächen auf Moosen, auf Sphagnum-Mooren (Mölder, Krasske). Nordisch-alpin.

\section{Eunotia tanensis A. Cleve}

Seltene Eunotia-Art, die Cleve-Euler (1934) aus Lappland bei Angeli am Oberlauf des Flusses Teno und Mölder (1937) aus Salla dargestellt hat. 


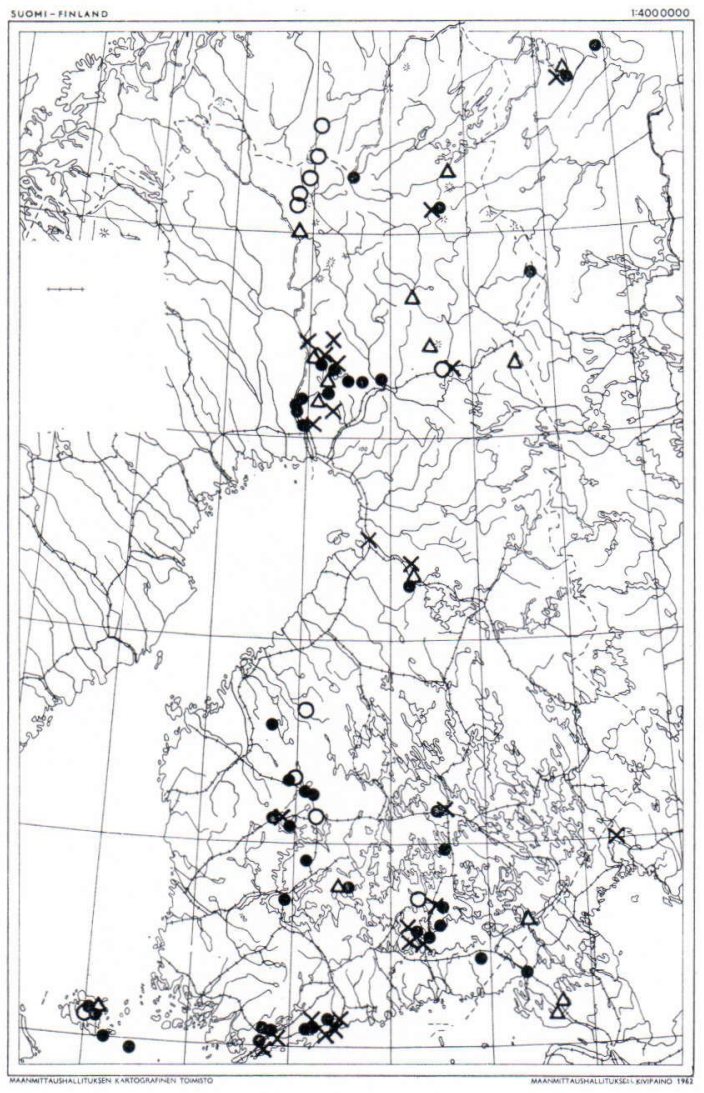

Abb. 9. Rezente Verbreitung von Eunotia tenella (Punkte), E. trinacria (Ringe), E. triodon (Dreiecke) und E. valida (Kreuze).

\section{Eunotia tenella (Grun.) Hustedt}

Kleine Eunotia-Art, die in Finnland häufig vorkommt. Wächst in Lachen, auf Mooren, feuchten Moosen und Felsflächen. Azidophile Form.

\section{Eunotia trinacria Krasske}

Kleine Eunotia-Art, in ihren Wuchsansprüchen ähnlich wie E. tenella.

Var. undulata Hustedt

Eine in Lappland an gewissen Stellen neben der Hauptart konstatierte Variation.

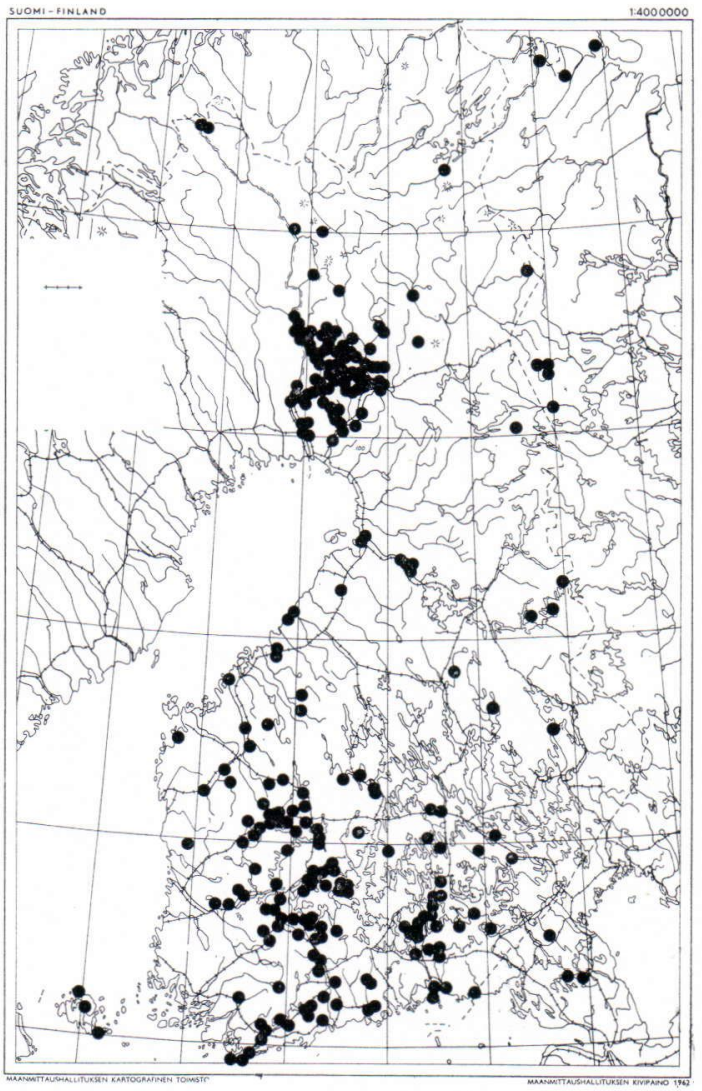

Abb. 10. Rezente Verbreitung von Eunotia veneris.

\section{Eunotia triodon Ehrenberg}

In Lappland verhältnismässig häufig in Weihern, Bächen und an feuchten Felswänden. Azidophile Form kühlen Wassers.

\section{Eunotia valida Hustedt}

Synonym: Eunotia grunowi var. limacida Å. Berg

In manchen Lachen und Mooren festgestellte Art.

Eunotia veneris (Kütz.) O. Müller

Synonyme: Eunotia incisa Greg., E. veneris var. incisa (Greg.) A. Cl.

Eine der häufigsten Eunotia-Arten in Finnland. Azidophile Form kleiner Gewässer. 
Var. exilis A. Cleve

Cleve-Euler (1953) hat eine kleine Variation, deren Enden vorwärts gekrümmt sind, vom Oberlauf des Tenojoki, aus Angeli, dargestellt.

Var. rhomboidea (Hustedt)

Unterscheidet sich von der Hauptart in erster Linie in Gürtelbandansicht, die rhomboid ist. Die Variation ist im allgemeinen mit der Hauptform vereinigt. Meriläinen (1969) hat sie (Eunotia rbomboidea) aus dem Valkiajärvi in Ruovesi dargestellt und mit Eunotia veneris verglichen.

\section{Actinella}

In der Schalenstruktur Eunotia ähnlich. In der Valvaransicht sind die Schalen gebogen, aber an dem einen Ende weiter. Transapikalstreifung und Raphen-Endknoten sowie an der Valvarebene die Randdörnchen sichtbar.

Actinella punctata Lewis

Seltene, nur in saurem, flachem Wasser $(\mathrm{pH}$ meist 4.5-6.5) epipytisch - u.a. an Blaualgen - wachsende Art. Erscheint oft in den gleichen Zusammenhängen wie Tabellaria binalis (Quennerstedt 1949, Kolbe 1950, Jørgensen 1953, Meriläinen 1967). 
TAFEL I

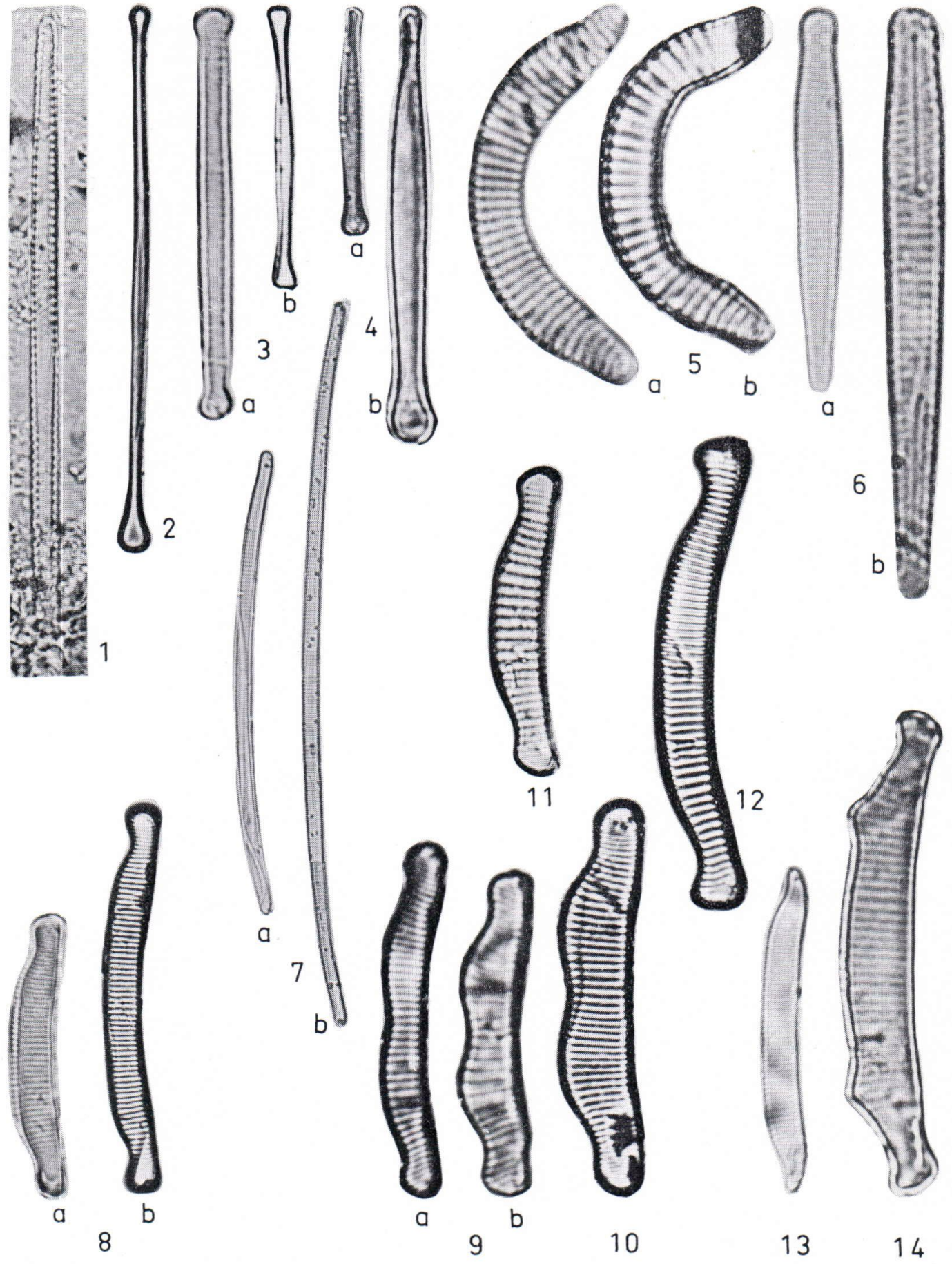

1. Thalassionema nitzschioides, 2. Asterionella formosa, 3. a-b. A. gracillima, 4. a-b. A. ralfsii, 5. a-b. Amphicampa hemicyclus, 6. a-b. Peronia beribandi, 7. a-b Eunotia olpina, 8. a-b E. arcus, 9. a-b E. a. var. bidens, 10. Übergangsform nach E. diodon, 11. E. arcus var. fallax, 12. E. a. var. uncinata, 13. E. attenuata, 14. E. bactriana. 
TAFEL II
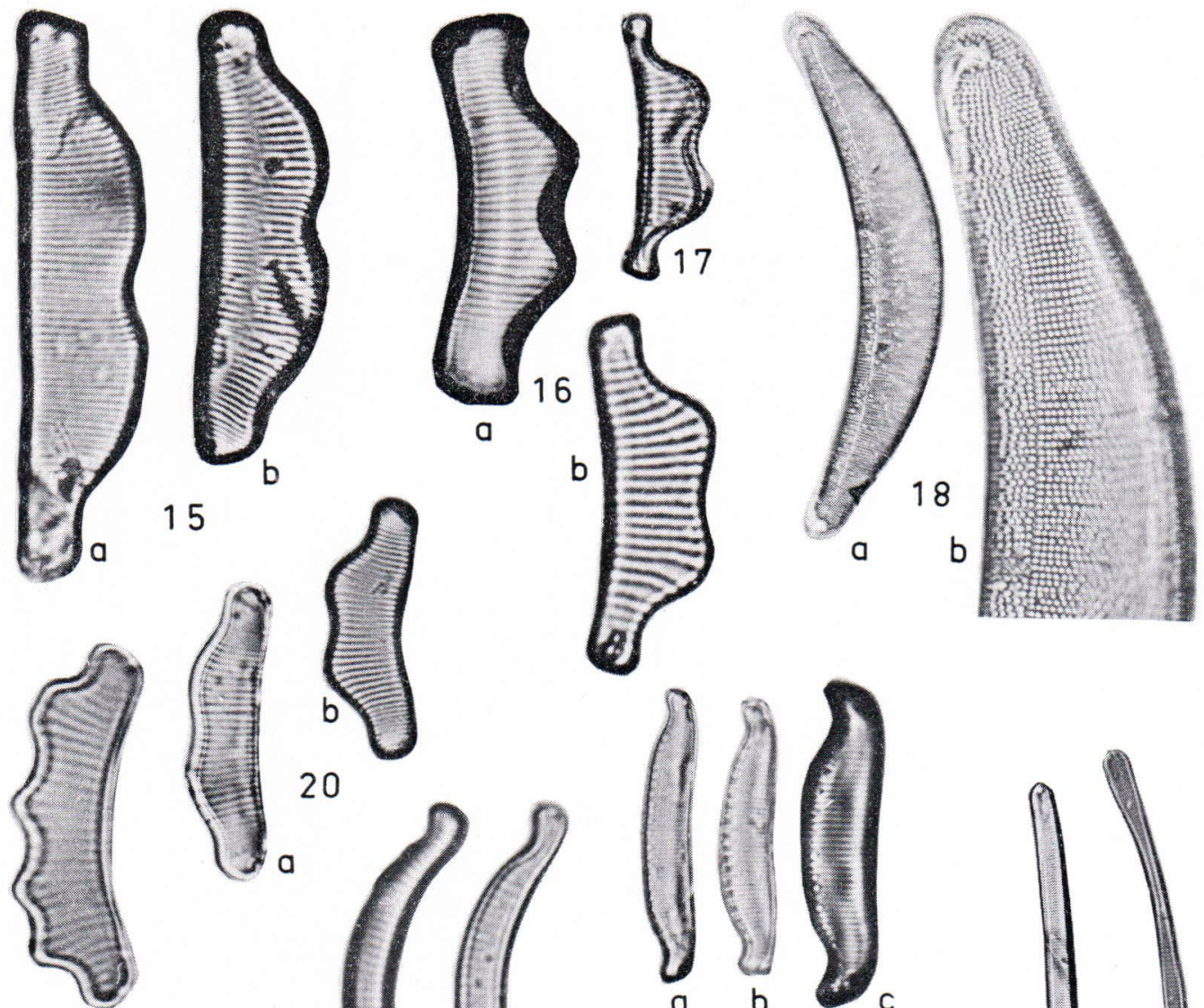

15
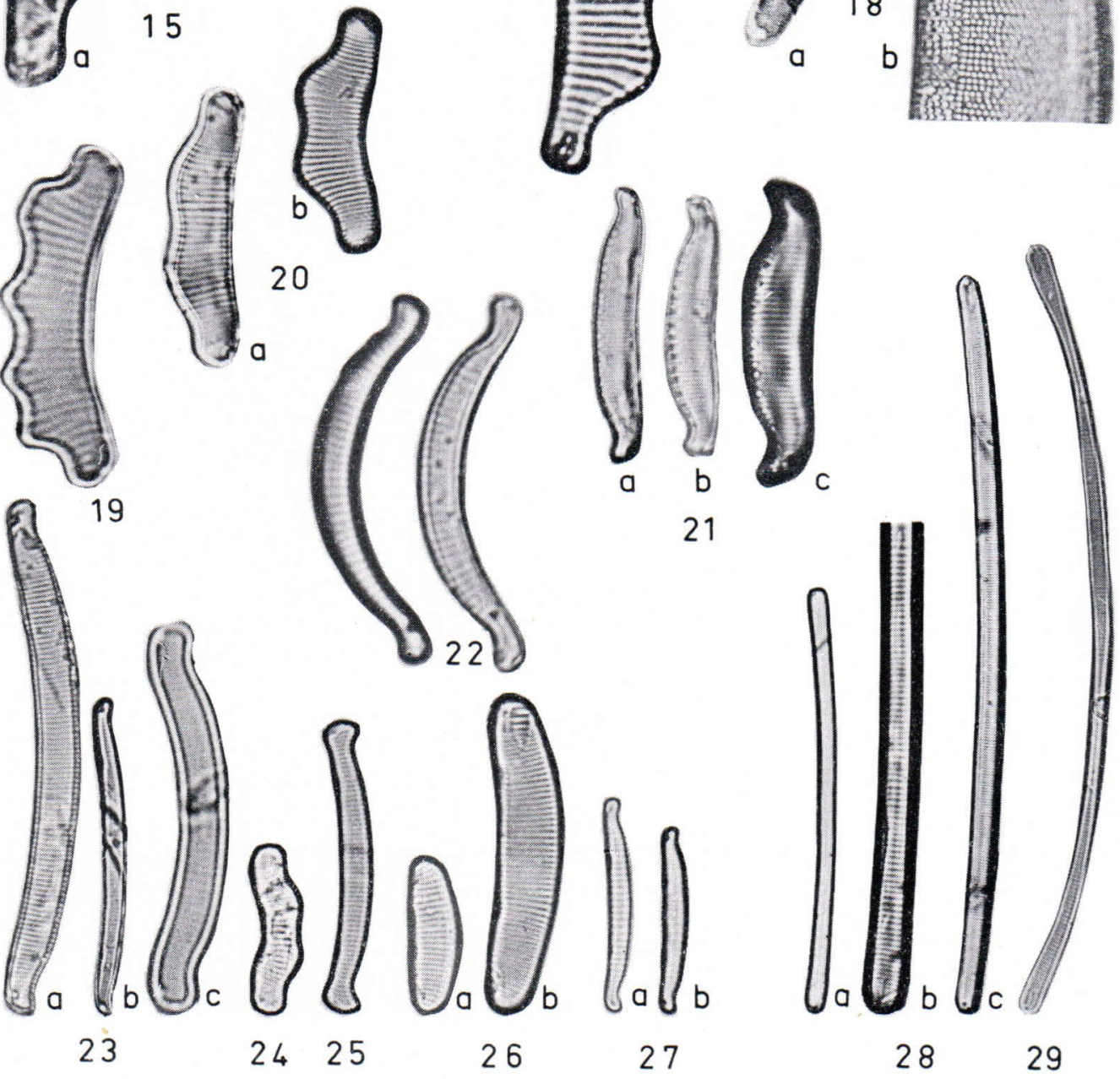

15. a-b Eunotia bidentula, 16. a-b E. bigibba, 17. E. b. var. pumila, 18. a-b E. clevei, 19. E. grista-galli, 20. a-b E. diodon, 21. a-c E. denticulata, 22. E. elegans, 23. a-c E. exigua, 24. E. e. var. bidens, 25. E. e. var. compacta, 26. a-b E. faba, 27. a-b E. fallax, 28. a-c E. flexuosa, 29. E. f. (var. pachycephala). 
TAFEL III
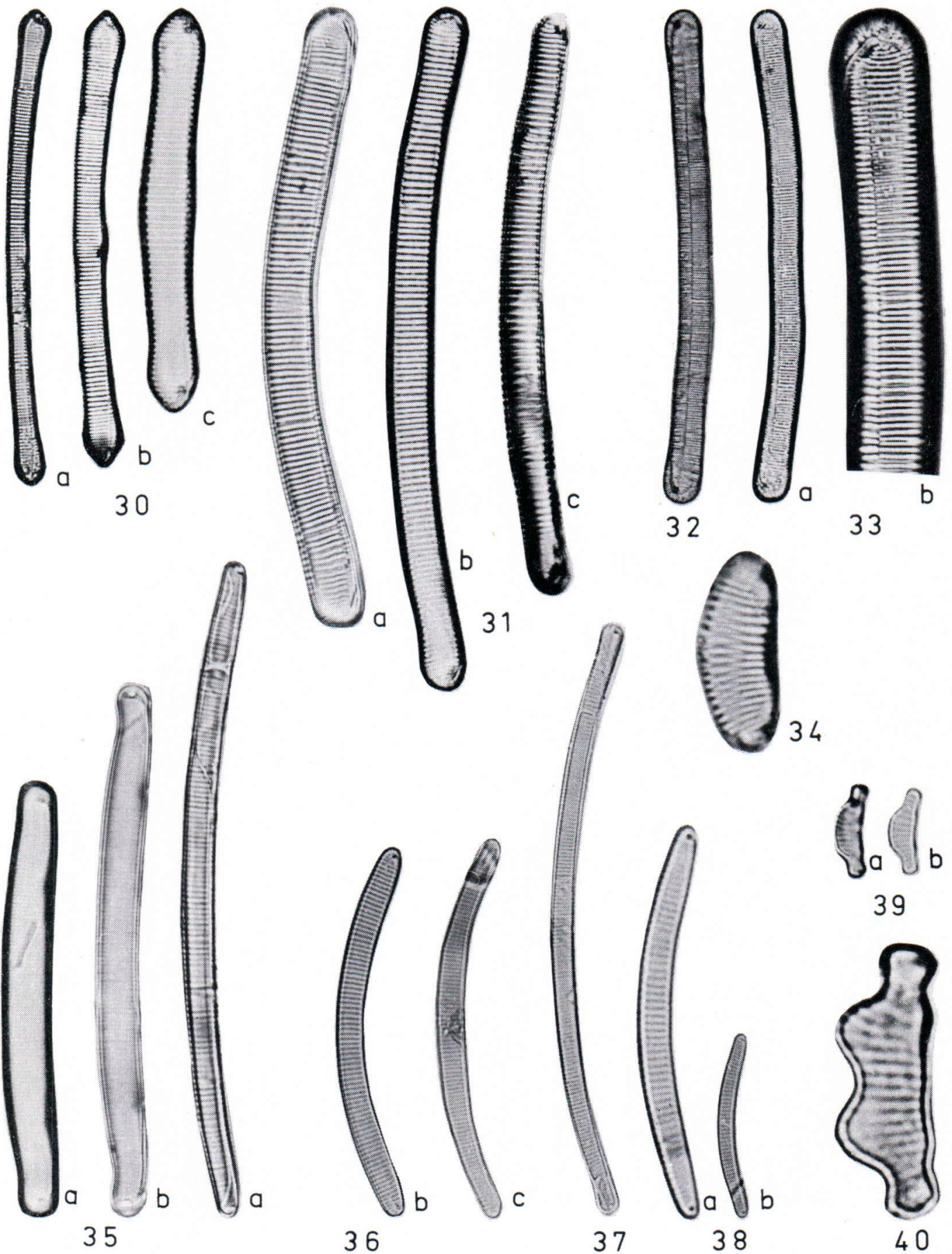

30. a-c Eunotia formica, 31. a E. gracilis var fennoscandica, 31. b-c E. gracilis, 32, 33. a-b E. hyberborea, 34. E. kncheliensis, 35. a-b E. lapponica, 36. a-c E. lunaris, 37. E. l. var. capitata, 38. a - b E. l. var. subarcuata, 39, a-b E. meisteri, 40. E. m. var. bidens. 


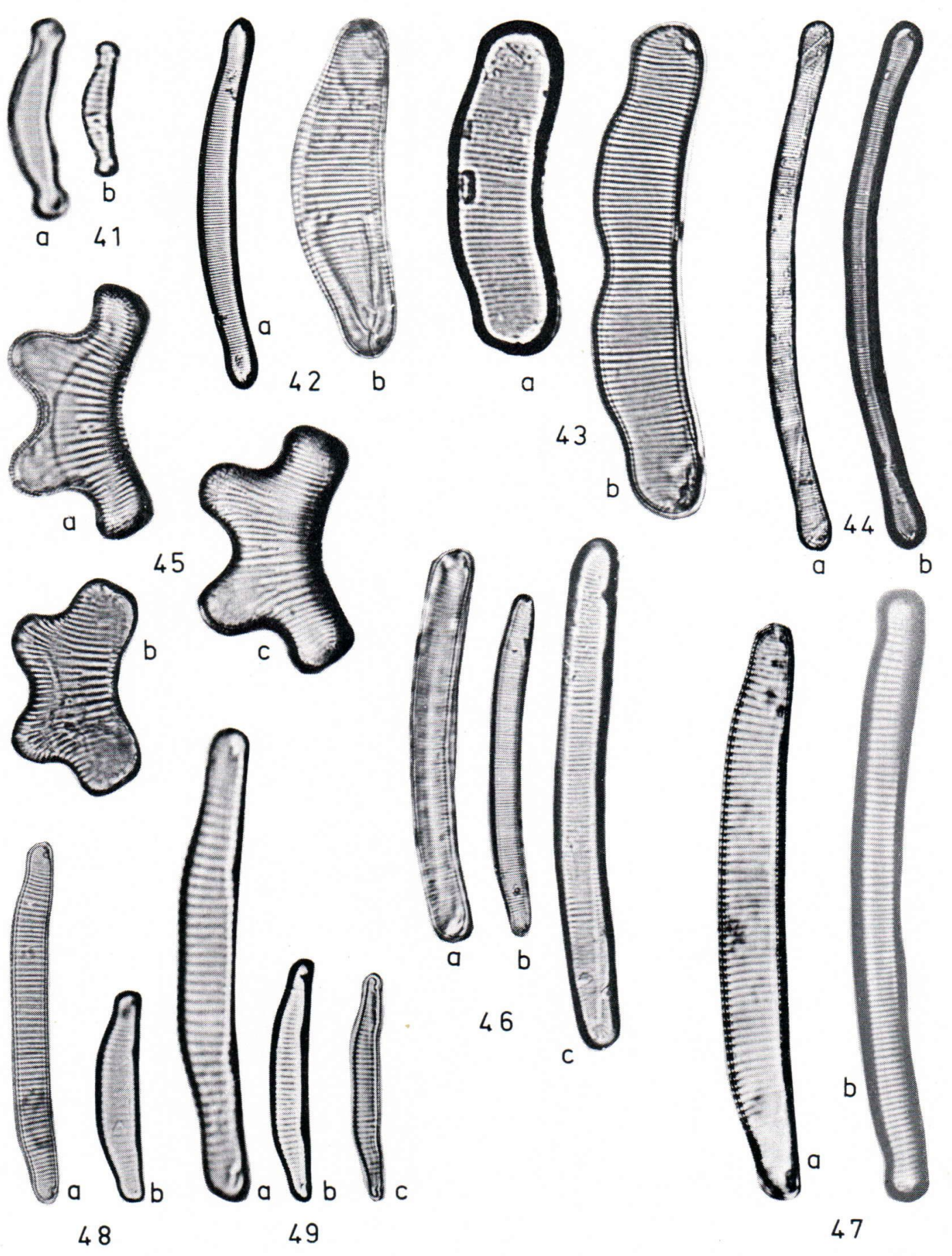

TAFEL IV

41. a-b Eunotia microcephala, 42. a-b E. monodom, 43. a Übergangsform nach var. bidens, 43. b E. m. var. bidens, 44. a-b E. m. var. maior, 45. a-c E. papilio, 46. a-c E. parallela, 47. a-b, 48. a E. pectinalis, 48. b E. p. var. minor, 49. a.-c E. p. var. minor fo. impressa. 
TAFEL V
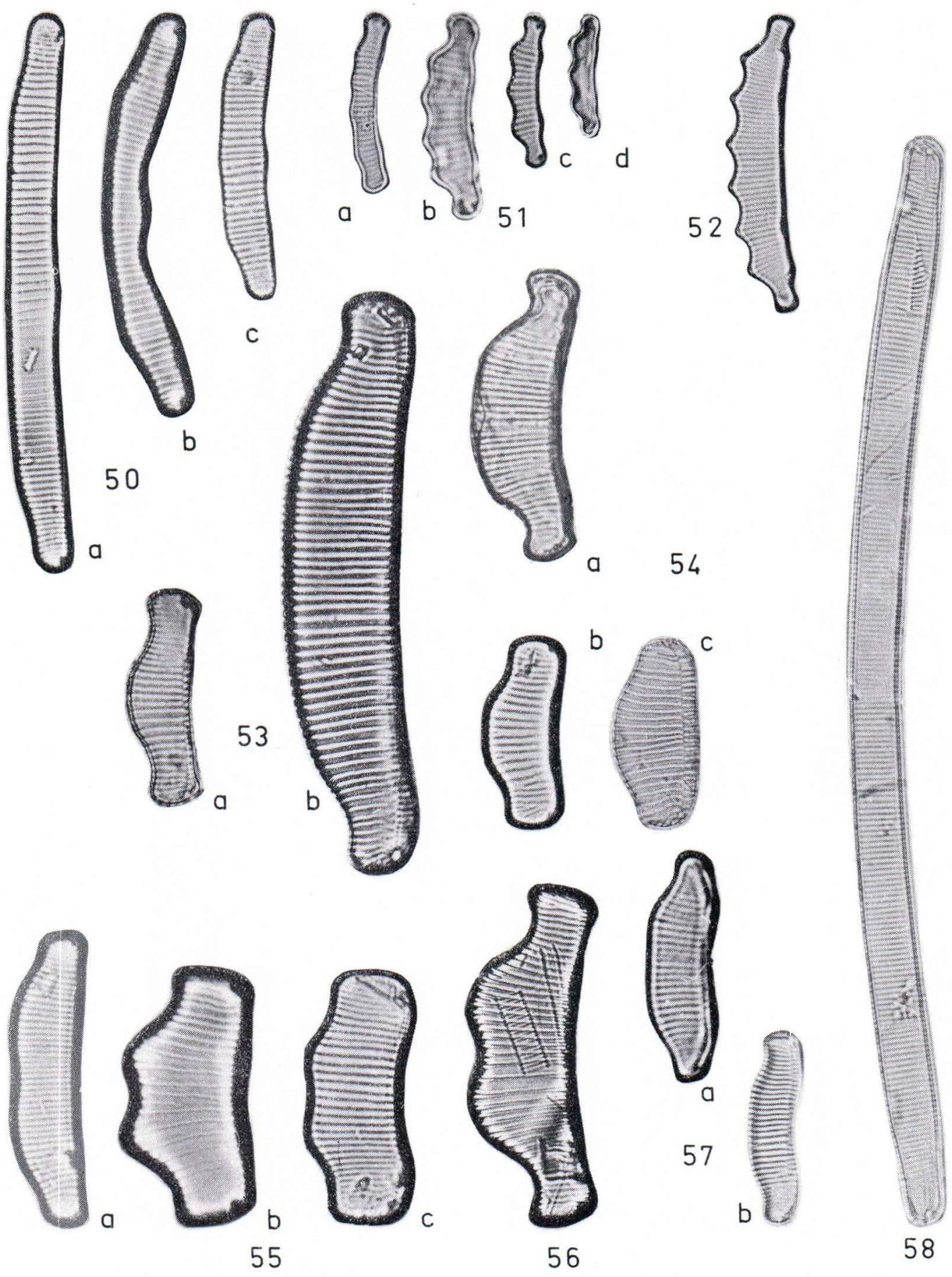

50. a-c Eunotia pectinalis var. ventralis, 51. a - d E. polydentula, 52. E. polyglyphis, 53. a-b. E. praerupta, 54. a-c E. p. var. inflata, 55. a-c E. p. var. bidens, 56. Übergangsform nach E. suecica, 57. a. E. praerupta, 57. b. E. p. var. muscicola, 58. E. pseudopectinalis. 

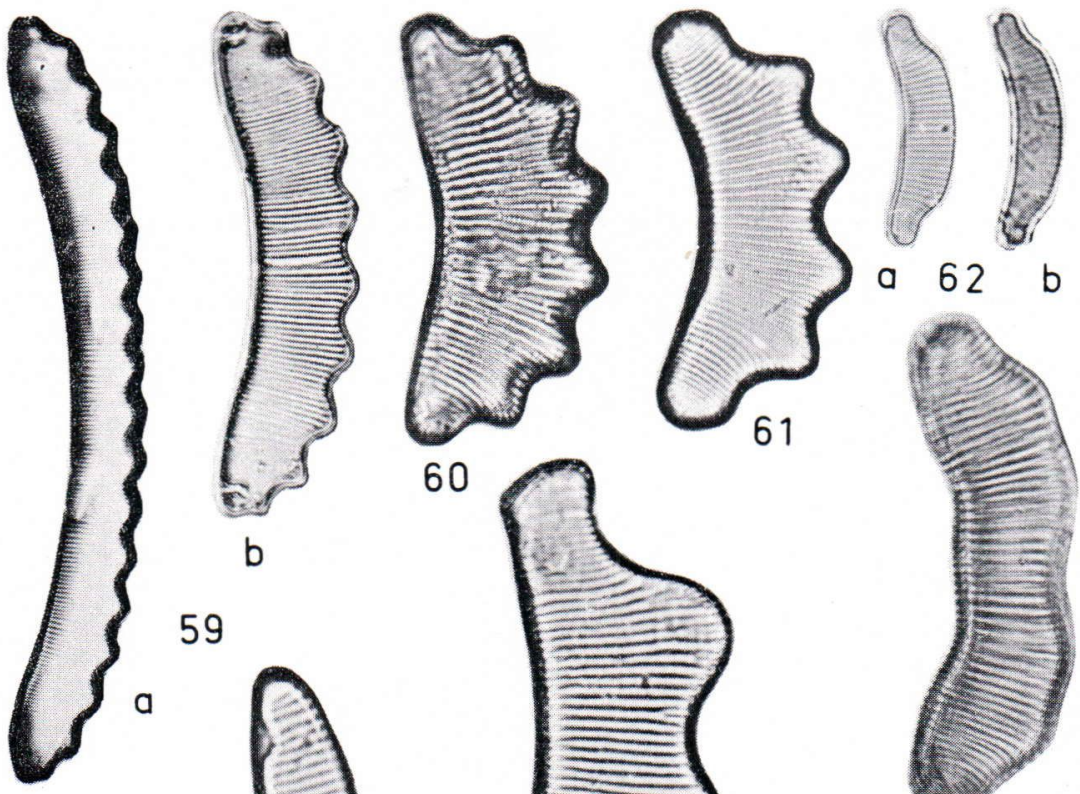

TAFEL VI
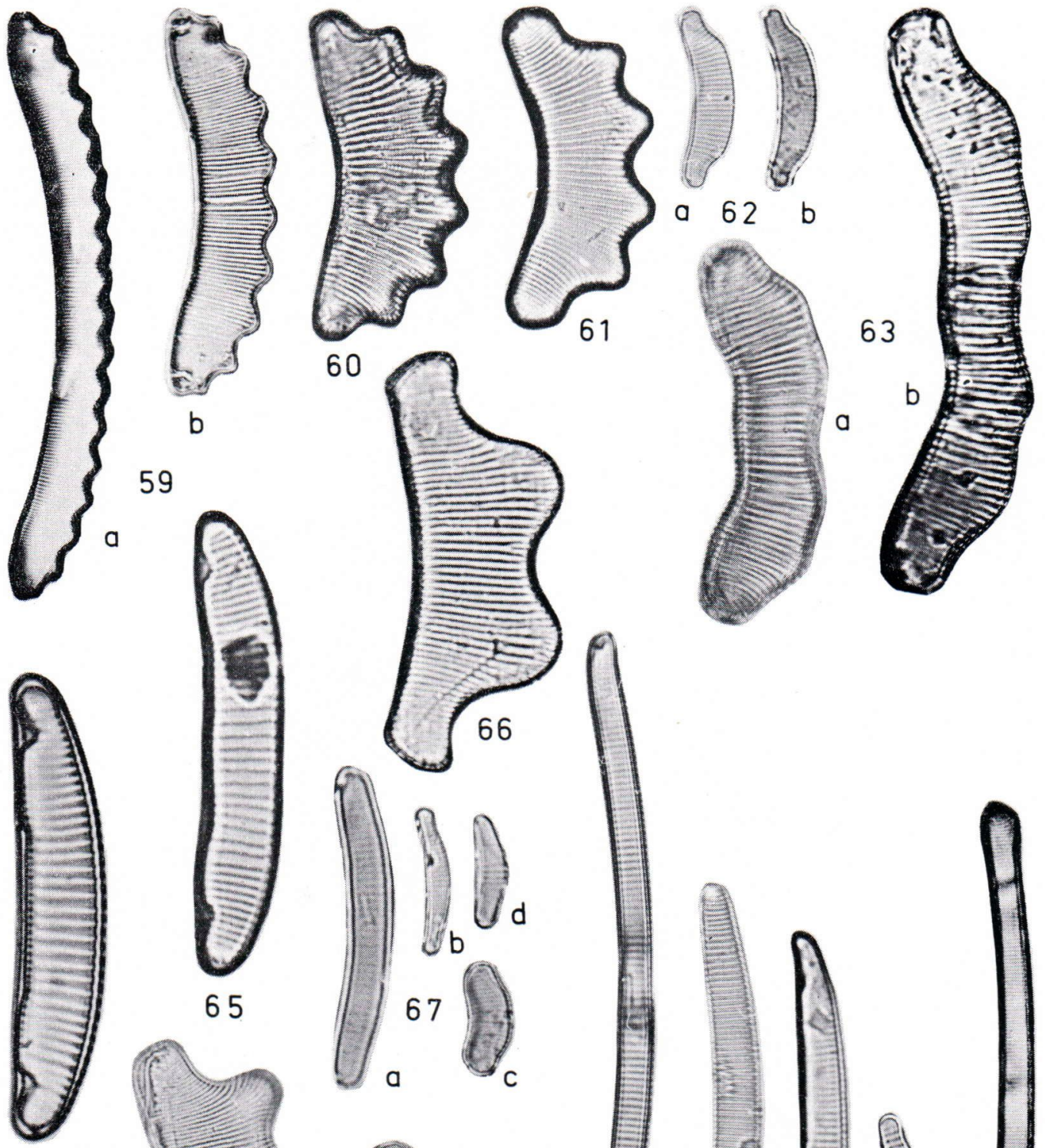

64

b

59
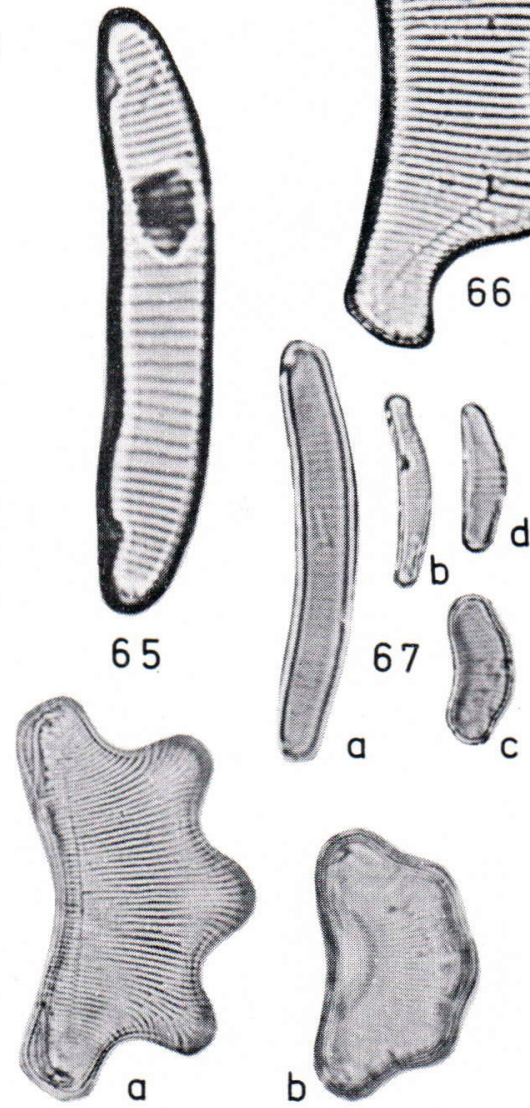

68
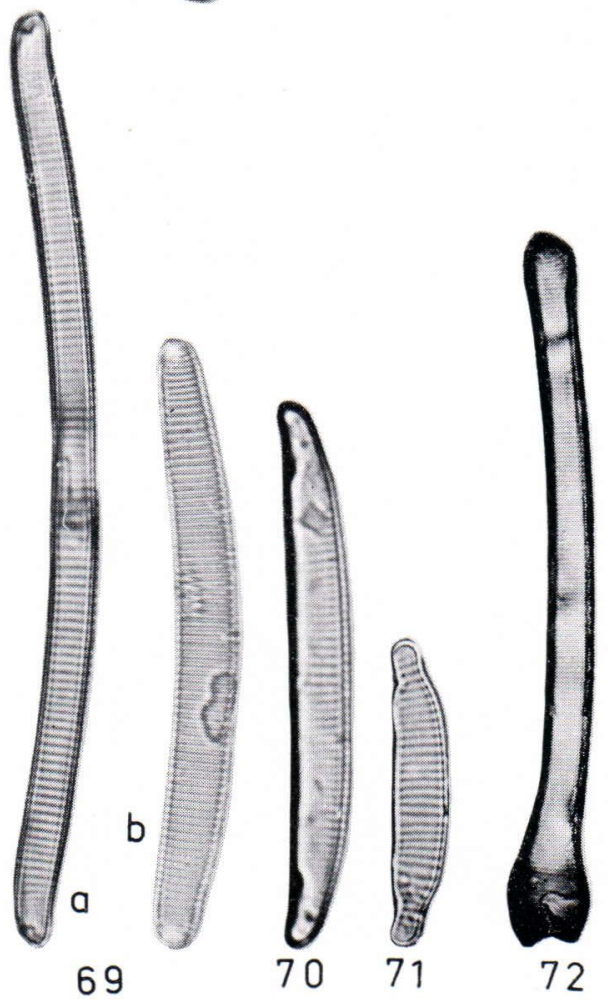

69

59. a-b Eunotia robusta, 60. E. $r$. var. diadema, 61. E. $r$. var. tetraodon, 62. a - b E. septentrionalis, 63. a-b E. sibiricas 64. E. sudetica, 65. E. s. var. bidens, 66. E. suecica, 67. a-d E. tenella, 68. a-b E. triodon, 69. a-b E. valida, $70,71$. E. veneris, 72. Actinella punctata. Photo E. Halme und R. Tynni. (etwa 1000 -fache Vergr., mit Ausnahme von Fig. 18 a, 28. a, c, 30. a-b, 31 a, 32, 33 a, 42. a, 44. a-b, 46. b, 59. a-b 400-fache; 41. a, 51. b 1 500-fache; 4. b, 5. a-b, 6. b, 14., 40. 2 000-fache.) 


\section{ERGÄNZENDE SCHRIFTEN}

Cholnoky, B. J. (1968) Die Ökologie der Diatomeen in Binnengewässern. National Institute for Water Research, Council for Scientific and Industrial Research, Pretoria, Südafrika, 3301 Lehre, Verl. J. Cramer.

- (1970) Bacillariophycées des Marais du Lac Bangweolo/ Bacillariophyceen aus den Bangweolo-Sümpfen. Exploration Hydrobiologique du bassin du Lac Bangweolo et du Luapula, Ed. J.-J. Symoens. Vol. V, fasc. 1. Bruxelles.

FogED, N. (1958) The diatoms in the basalt area and adjoining areas of archean rock in West Greenland Meddel. Grønland 156 (4): 1-146.

Jørgensen, Erik. G. (1953) The diatoms Tabellaria binalis and Actinella punctata in South-Norway. Nytt Magasin for Botanikk. Oslo.
Kolbe, R. W. (1948) Einige bemerkenswerte Diatomeen aus schwedischen Gewässern. Sv. Bot. Tidsk. 42. Uppsala.

- (1950) Über rezente Standorte von Actinella punctata Lew. in Skandinavie. Sv. Bot. Tidsk. 44, 1. Uppsala.

Mackeraeth, F. J. (1953) Phosphorus utilization by Asterionella formosa Hass. Journal of Experimental Botany, Vol. 4: 296-313.

Merilärnen, Jouko (1969) The diatoms of the meromictic Lake Valkiajärvi, in the Finnish Lake District. Ann. Bot. Fenn. 6: 77-104.

Patrick, Ruth (1940) Some new diatoms from Brazil. Notulae Naturae 59. Philadelphia.

Pork, Mara (1970) On the ecology of diatoms in Estonia Lakes. (in Russisch). Transactions of the Tartu State University. Papers of Botany. Tartu. 